\title{
Progressive Scleral Necrosis following l-125 Plaque Radiotherapy for Ciliochoroidal Melanoma with Protruding Extraocular Mass
}

\author{
Jordan R. Hill Zélia M. Corrêa \\ Department of Ophthalmology, College of Medicine, University of Cincinnati, Cincinnati, Ohio, USA
}

\section{Key Words}

Scleral necrosis $\cdot$ I-125 plaque radiotherapy $\cdot$ Choroidal melanoma

\begin{abstract}
Purpose: The aim of this study was to describe the side effects of I-125 brachytherapy in the treatment of uveal melanoma. Methods: This study was conducted as a case report. Results: We report a case of scleral necrosis and protruding episcleral mass following the treatment of uveal melanoma with I-125 brachytherapy. Conclusions: Scleral necrosis after plaque radiotherapy can clinically simulate tumor recurrence with extraocular extension. The management of uveal melanoma requires a careful clinical follow-up, weighing the implications of treatment morbidity and mortality.
\end{abstract}

(c) 2015 S. Karger AG, Basel

\section{Introduction}

Eye-preserving treatments are usually preferred for patients with medium-sized posterior uveal melanoma of a nonjuxtapapillary location, since there is no survival difference between patients treated with radiation versus enucleation [1]. However, radiation treatment may have an impact on patient morbidity due to its side effects including cataract, retinopathy, maculopathy, and less commonly, scleral necrosis [2]. In this report, we describe a patient with a ciliochoroidal melanoma treated with I-125 plaque radiotherapy who later developed progressive scleral necrosis and a protruding episcleral mass, raising the concern for recurrent melanoma.

\section{Case Report}

A 62-year-old male complaining of decreased vision presented a large ciliochoroidal melanocytic tumor OS with associated exudative retinal detachment (fig. 1a). Initial visual acuity was 20/ $20^{-2}$ OD and $20 / 80^{-1}$ OS. B-scan ultrasound showed a ciliochoroidal tumor OS, extending from the ciliary body into the choroid superotemporally with limited scleral invasion measuring 11.4 $\mathrm{mm}$ in thickness (fig. $1 \mathrm{~b}$ ) and $16 \mathrm{~mm}$ in basal diameter. The tumor's TNM staging, as per the AJCC staging system, was $\mathrm{T}_{3 \mathrm{~b}} \mathrm{~N}_{0} \mathrm{M}_{0}$.

After discussing management options, the patient chose I-125 plaque radiotherapy to treat the tumor and prognostic fine-needle aspiration biopsy at the time of plaque implantation. A $21-\mathrm{mm}$ diameter COMS plaque delivered 80 Gy to the tumor apex and 732 Gy to the sclera over a 4-day period. Fine-needle aspiration biopsy showed a malignant melanoma with mixed spindle and epithelioid cell type but no scleral necrosis.

The tumor subsequently regressed significantly, but his vision remained poor. Twenty-three months after I-125 plaque treatment, the patient's visual acuity was no light perception OS with extensive pupillary synechia, iris neovascularization, posterior subcapsular cataract, and a small, dark-gray flat scleral spot $6.5 \mathrm{~mm}$ from the limbus initially thought to be localized scleral thinning (fig. 1c). Bscan ultrasound showed further decrease in tumor thickness to 1.8 $\mathrm{mm}$ (fig. 1d). The scleral spot remained stable until 52 months after

\section{KARGER}

E-Mail karger@karger.com

www.karger.com/oop (c) 2015 S. Karger AG, Base

2296-4681/15/0023-0136\$39.50/0
Zélia M. Corrêa, MD, $\mathrm{PhD}$

Department of Ophthalmology, College of Medicine, University of Cincinnat Medical Arts Building, Suite 1500

Cincinnati, OH 45219 (USA)

E-Mail correazm@uc.edu 

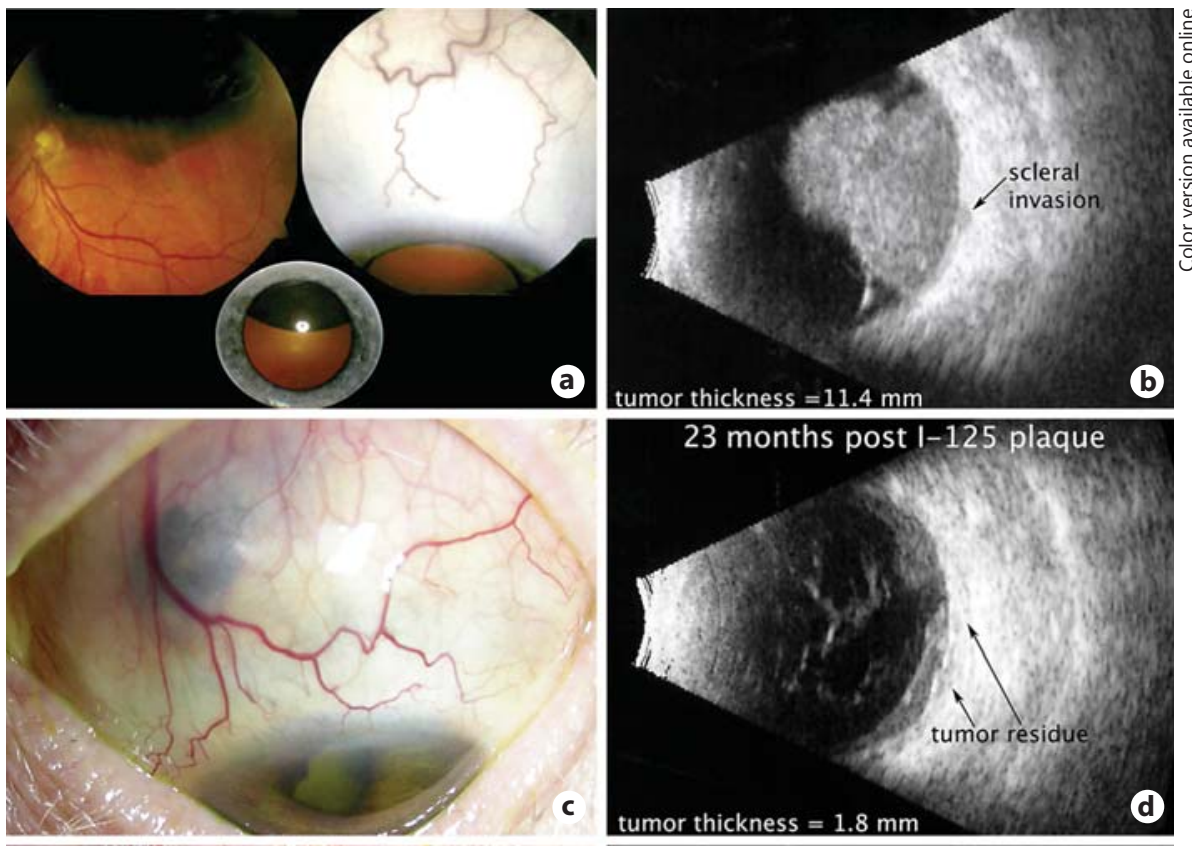

Fig. 1. Clinical course of the reported patient. a Fundus photograph at baseline, showing a large ciliochoroidal tumor centered at the equator superiorly. Observe the clinically intact sclera superiorly and the tumor seen through the pupil, partially obstructing the visual axis (small image). b Baseline B-scan, showing a ciliochoroidal mass with an eccentric cross-sectional mushroom shape and limited scleral invasion (arrow). c External photograph, showing a dark episcleral nodule superonasally. d B-scan 23 months after radiotherapy, showing the shrunken ciliochoroidal melanoma and tumor residue (arrows). e External photograph, showing increased nodularity, suggestive of extraocular extension of ciliochoroidal melanoma. f B-scan 52 months after radiotherapy, showing stable tumor and the area of extraocular extension, indicated by arrows and labeled EOE.
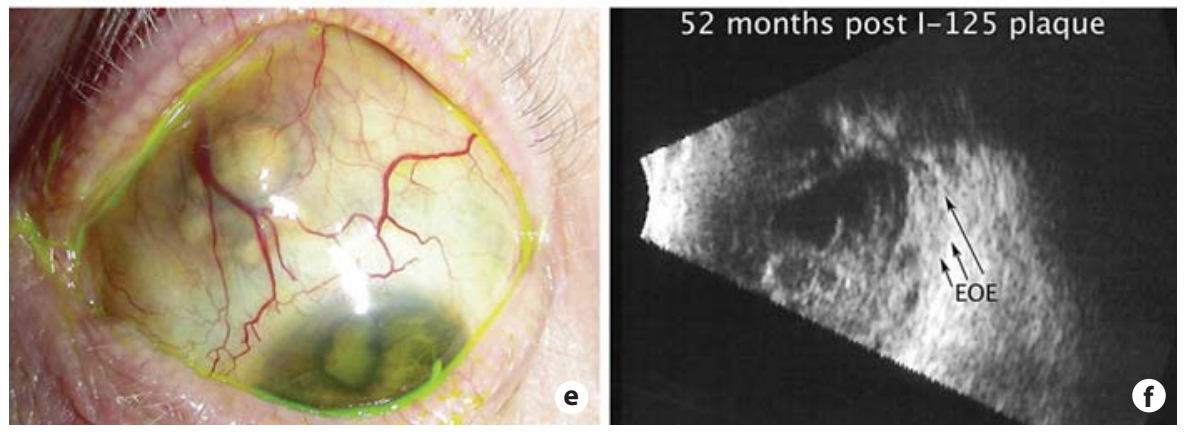

brachytherapy when it progressed to a distinctively nodular protruding lesion (fig. 1e). B-scan ultrasound showed a relatively stable intraocular tumor (fig. 1f), raising our concern for local tumor relapse and possible extraocular tumor extension. After explaining the potential risk of tumor recurrence versus the status of the eye, the patient agreed to have enucleation OS.

Gross examination of the enucleated globe revealed massive bleeding, resulting in a brown staining of intraocular structures, retinal detachment, neovascularization of the iris, cataract, and a regressed solid ciliochoroidal mass protruding through the sclera (fig. 2a, b). Histopathology showed focal, full-thickness scleral necrosis superonasally, overlying a necrotic mass with patchy chronic, nongranulomatous inflammation, but no evident vasculitis (fig. 2c, f). The extruded, nodular tissue consisted mainly of organized cholesterol clefts and exudative material with pigment granules and rare macrophages (fig. 2d). The irradiated tumor was composed mainly of small pyknotic cells with foamy cytoplasm and rare morphologically intact spindle and epithelioid melanoma cells (fig. 2e). The vitreous was dense and filled with proteinaceous material, and the retina was mostly atrophic and gliotic. Although a few viable appearing tumor cells were present within the sclera, no definite tumor cells were extending outside the sclera.

Progressive Scleral Necrosis after Plaque for Ciliochoroidal Melanoma

\section{Discussion}

Our group has previously reported a patient with ciliochoroidal melanoma treated by I-125 plaque radiotherapy that developed scleral necrosis 1 month after treatment [3]. Nevertheless, the patient reported herein developed a scleral lesion approximately 2 years after brachytherapy. While scleral necrosis has been reported after plaque radiotherapy treatment for choroidal melanoma, it is relatively rare $(1 \%)$ in patients treated specifically with I-125 [2].

The diagnosis of scleral necrosis is mainly clinical [4], and its course can be unpredictable [2]. Thus, distinguishing extraocular tumor extension after radiation from severe progression of scleral necrosis can be challenging.

The exact pathophysiology of scleral necrosis is unknown, but several mechanisms have been proposed. Although the patient received the recommended dose of 

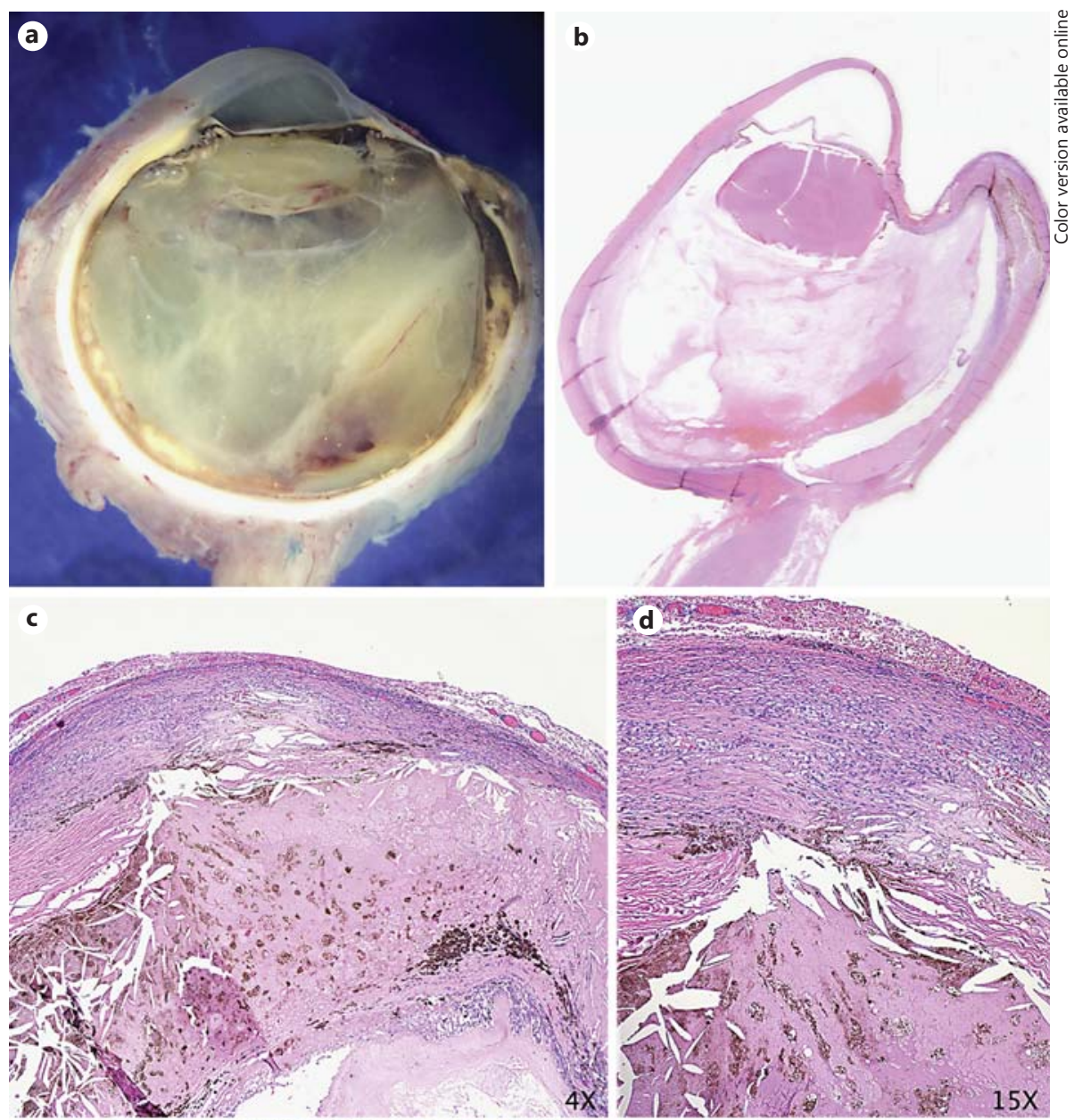

Fig. 2. Gross specimen and histopathological findings. a Gross specimen reveals dense, organized and partially reabsorbed vitreous hemorrhage with brown staining of intraocular structures, retinal detachment, neovascularization of the iris, cataract, and a regressed solid ciliochoroidal mass protruding through the sclera (as seen on the right side of the globe). $\mathbf{b}$ Lowresolution photograph of the microslide provides a comparative view of the gross specimen. c Focal scleral necrosis with protruding acellular material (and cholesterol clefts). HE. $\times 4$. d Detail of focal scleral necrosis. HE. $\times 15$. e Microphotograph showing the pyknotic cells with foamy cytoplasm. Rare intact tumor cells are observed on the upper right corner. HE. $\times 30$. f Lowresolution photomicrograph, showing pockets of nongranulomatous inflammation in the sclera and choroid. HE. $\times 6$.
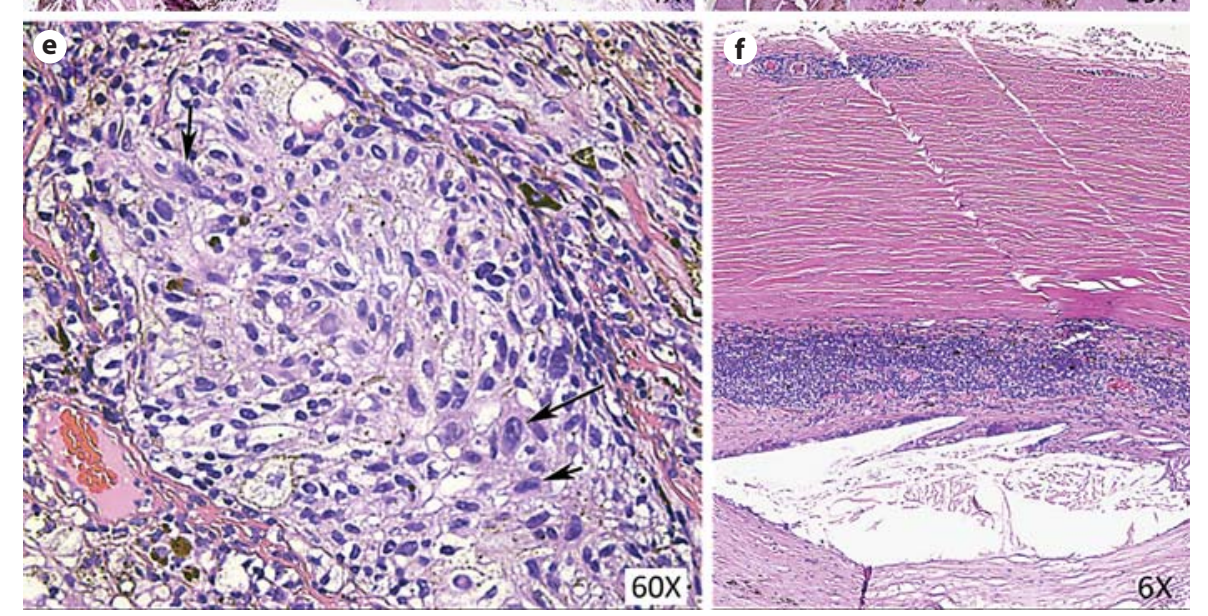

radiation to the sclera, scleral necrosis has been reported to occur after radiation doses of $\geq 400 \mathrm{~Gy}$ [2] to the sclera and $88.3 \mathrm{~Gy}$ to the tumor base [4]. Other possibilities that could not be entirely excluded were surgically induced local ischemia, granulomatous inflammation from suture material at the time of plaque placement, or scleral perforation during plaque implantation or extraocular muscle disinsertion/reinsertion. Another possible etiology is secondary occult vasculitis caused by infectious or systemic inflammatory disorders. The timing 
of scleral necrosis resulting from radiotherapy has also been noted to have a mean onset of 32 months after radiotherapy.

Our case features an unusual presentation of scleral necrosis after plaque radiotherapy, simulating tumor recurrence with extraocular extension. The management of uveal melanoma requires a careful clinical follow-up, weighing the implications of treatment morbidity and mortality.

\section{Acknowledgements}

We would like to thank Drs. James Augsburger and Bradley Huth for their expert participation in the care of this patient.

\section{Statement of Ethics}

The patient described herein gave the authors written consent to report this case, granted personal identifiers were omitted.

\section{Disclosure Statement}

The authors declare that they have no conflicts of interest to disclose.

\section{References}

Progressive Scleral Necrosis after Plaque for Ciliochoroidal Melanoma
1 Collaborative Ocular Melanoma Study Group: The COMS randomized trial of Iodine $\mathrm{e}^{125}$ brachytherapy for choroidal melanoma. V. Twelve-year mortality rates and prognostic factors: COMS Report No. 28. Arch Ophthalmol 2006;124:1684-1693.

2 Kaliki S, Shields CL, Rojanaporn D, et al: Scleral necrosis after plaque radiotherapy of uveal melanoma: a case-control study. Ophthalmology 2013;120:1004-1011.
3 Corrêa Z, Augsburger JJ, Freire J, Eagle RC: Early-onset scleral necrosis after Iodine I 125 plaque radiotherapy for ciliochoroidal melanoma. Arch Ophthalmol 1999;117:259-261.

4 Radin PP, Lumbroso-LeRouic L, Levy-Gabriel C, Dendale R, Sastre X, Desjardins L: Scleral necrosis after radiation therapy for uveal melanomas: report of 23 cases. Graefes Arch Clin Exp Ophthalmol 2008;246:1731-1736. 